\title{
A 64-year-old man admitted to the Emergency Department with an unusual case of intestinal obstruction: decision-making in the Emergency Department
}

\author{
Jessica Attene - Giuseppe Pepe $\cdot$ Simone Vanni $\cdot$ Melisenda Chiarlone $\cdot$ \\ Beatrice Dilaghi $\cdot$ Stefano Grifoni
}

Received: 5 February 2008/Accepted: 8 March 2008/Published online: 25 June 2008 (C) SIMI 2008

\section{Presentation and history}

Dr. J. Attene: A 64-year-old man was transported from his home to the Emergency Department (ED) because of a sudden worsening of abdominal pain and vomiting, on 15 February 2007.

On admission he denied fever, chest pain, shortness of breath, diarrhea or urinary symptoms. The abdominal pain was localized in the upper abdominal quadrants and did not radiate to the back or arms. There was no history of passing blood per rectally. Passage of flatus and feces was absent in the prior $24 \mathrm{~h}$.

The patient had been in good health before admission, did not have a history of previous abdominal pain or exposure to patients with gastroenteritis, and there was no family history of inflammatory bowel disease. He usually smoked cigarettes and drank alcohol ( $1 \mathrm{~L}$ of wine per day), but denied recreational drug use. Is there any question regarding the history?

Dr. S. Vanni: Did you have any information about other significant medical or surgical history, or past recovery? In particular, did he undergo invasive surgical operations?

Dr. J. Attene, Dr. G. Pepe: The patient had mild hypertension, not treated. There was no history of diabetes or previous cerebrovascular or cardiovascular events. The past surgical history consisted of an appendectomy in adolescence, and a laparotomy performed 48 years ago, but

J. Attene $\cdot$ G. Pepe $\cdot$ S. Vanni $\cdot$ M. Chiarlone

B. Dilaghi $(\varangle) \cdot$ S. Grifoni

Department of Emergency Medicine,

Azienda Ospedaliera-Universitaria Careggi,

University of Florence, Viale Morgagni 85,

50134 Florence, Italy

e-mail: beatrice.dilaghi@tin.it on the admission he was unable to describe the exact reason, except reporting something about gastric peptic ulcer.

On physical examination, vital signs upon arrival were a tympanic temperature of $37.0^{\circ} \mathrm{C}$, heart rate 120 beats/min, blood pressure $200 / 115 \mathrm{mmHg}$, respiratory rate 22 breaths/ min, and an oxygen saturation of $98 \%$ on room air. The patient was awake and communicative although he appeared to be suffering and dehydrated. The heart examination revealed tachycardia. The abdomen exhibited diffused tenderness, Blumberg sign was absent, there were no other signs of peritoneal irritation, and peristaltic sounds were present. The hernia orifices were normal. The extremities were without edema, with good peripheral pulses. The skin examination did not show rash or other alterations. The electrocardiogram showed sinus tachycardia (Fig. 1). Laboratory tests were performed (Table 1). The arterial blood gas revealed a normal $\mathrm{pH}$ with hypocapnia, elevated levels of serum lactate $(4.3 \mathrm{mmol} / \mathrm{L})$ and a low $\mathrm{HCO}_{3}{ }^{-}(19.6 \mathrm{mmol} / \mathrm{L})$. Laboratory data showed leukocytosis $\left(21,970 / \mathrm{mm}^{3}\right)$, elevated levels of blood sugar, lactate dehydrogenase, creatine kinase and hemoglobin with a hematocrit of $55.1 \%$ and normal erythrocyte count.

A nasogastric tube was placed to low suction, and about $3,000 \mathrm{~mL}$ of bloodstained fluid were drained. Because of prolonged vomiting and obvious dehydration, an adequate fluid replacement was started, and because of concern for a bacterial infection, wide-spectrum antibiotics (combination of amoxicillin and clavulanic acid $2.2 \mathrm{~g}$ ) were administered as well. An abdominal X-ray series and ultrasonography of the abdomen were performed, but no pathological findings were revealed.

Dr. S. Grifoni: Abdominal pain accounts for over five million visits annually to Emergency Departments in United States [1]. Between 20 and $40 \%$ of these patients 


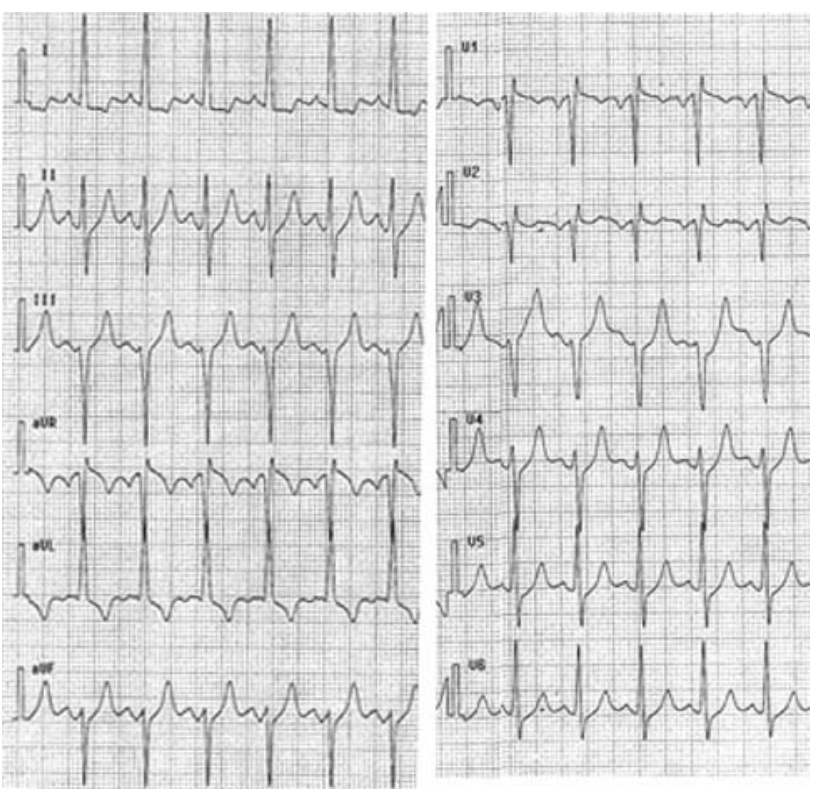

Fig. 1 Electrocardiogram at the admission showing sinus tachycardia

Table 1 Results of laboratory tests ${ }^{\mathrm{a}}$

\begin{tabular}{|c|c|c|c|}
\hline Variable & Admission & After $3 \mathrm{~h}$ & Normal range \\
\hline Glucose (mg/dL) & 302 & 220 & $65-110$ \\
\hline AST GOT (U/L) & 41 & 29 & $5-40$ \\
\hline ALT GPT (U/L) & 35 & 24 & $5-40$ \\
\hline Creatinine (mg/dL) & 1.8 & 1.7 & $0.6-1.5$ \\
\hline Urea $(\mathrm{g} / \mathrm{dL})$ & 0.63 & 0.66 & $0.1-0.5$ \\
\hline Sodium (mEq/L) & 133 & 140 & $135-146$ \\
\hline Potassium (mEq/L) & 3.8 & 4.5 & $3.5-5.3$ \\
\hline Chloride (mEq/L) & 97 & 106 & $98-110$ \\
\hline Creatine kinase (U/L) & 333 & 219 & $20-160$ \\
\hline Myoglobin (ng/mL) & 284 & 234 & \\
\hline Cardiac troponin I $(\mathrm{ng} / \mathrm{mL})$ & 0.07 & 0.06 & $0-0.15$ \\
\hline Total bilirubin (mg/dL) & 1.20 & 0.69 & $0.3-1$ \\
\hline Lactate dehydrogenase (U/L) & 423 & 356 & $120-270$ \\
\hline Amylase (U/L) & 61 & 44 & $25-125$ \\
\hline White-cell count $\left(\times 10^{9} / \mathrm{L}\right)$ & 21.97 & 15.62 & $4-10$ \\
\hline $\mathrm{RBC}$ count $\left(\times 10^{12} / \mathrm{L}\right)$ & 5.98 & 5.14 & $4.5-6.1$ \\
\hline Hemoglobin $(\mathrm{g} / \mathrm{dL})$ & 19.3 & 16.5 & $14-18$ \\
\hline Hematocrit; Hct (\%) & 55.1 & 48.5 & $42-52$ \\
\hline MCV (fL) & 92.1 & 94.4 & $81-94$ \\
\hline Platelet count $\left(\times 10^{12} / \mathrm{L}\right)$ & 234 & 174 & $140-440$ \\
\hline Prothrombin activity $(\%)$ & 117 & 97 & $70-130$ \\
\hline I.N.R. & 0.94 & 1.02 & $0.8-1.2$ \\
\hline APTT (s) & 30.8 & 27.4 & $38-40$ \\
\hline Fibrinogen (mg/dL) & 441 & 313 & $200-450$ \\
\hline
\end{tabular}

${ }^{a}$ To convert the values for glucose to millimoles per liter, multiply by 0.05551

$R B C$ Red blood cell, $M C V$ mean corpuscular volume, APTT partialthromboplastin time will require inpatient admission. In our ED, abdominal pain was the main complaint in about $16 \%$ of incoming patients (7,500 cases/year) of which $24 \%$ required hospital admission. Physical examination and laboratory evaluation can both lack sensitivity, hence it is often difficult to assess the etiology of abdominal pain during the course of the ED visit.

In our case we have a patient presenting to the ED with worsening vomiting and abdominal pain. Clinical data suggested the diagnosis of an acute intestinal obstruction with evident fluid depletion due to vomiting and fluid sequestration in the bowel. However, abdominal $\mathrm{X}$-ray series and ultrasonography of abdomen did not show any specific abnormalities. What was your decisionmaking process at this point? Did you ask for a surgery consult?

Dr. G. Pepe: A complete past pathological history revaluation was obtained from a family member, who had arrived urgently in the ED. New information revealed a Billroth II anastomosis for gastroduodenal peptic ulcer 48 years ago. A surgery consult was promptly requested but despite the typical clinical presentation suggesting an acute intestinal obstruction, the surgeons wished to obtain a computed tomographic (CT scan) of the abdomen with intravenous contrast to understand the cause of the obstruction.

Dr. M. Chiarlone: The CT scan has become a useful method in diagnosing the presence and the cause of intestinal obstruction, especially in cases where clinical and plain film findings are equivocal. The plain abdominal radiograph remains the current first-line radiological test in suspected bowel obstruction, however there are documented failings of the performance of plain films especially in low grade or early obstruction. The true value of the CT scan in the investigation of these patients is not to make diagnosis of obstruction per se but to define the cause and the severity of the obstruction and thereby influence management. Studies demonstrate that a CT scan is able to establish the cause of obstruction correctly in $73-95 \%$ of cases. May we examine the radiographic findings?

Dr. B. Dilaghi: The CT scan showed dilated loops of fluid-filled small bowel with a few air-fluid levels (Figs. 2, 3), a dilated gallbladder, without stones, and a nondilated biliary tract. No alteration of the bowel wall was demonstrated. There were multiple focal splenic lesions thought to be splenic infarcts. A small adrenal mass of $15 \mathrm{~mm}$ was discovered in the left adrenal gland. The CT scan confirmed the hypothesis of intestinal obstruction, suggesting the involvement of small bowel, but did not detect any clear cause of obstruction. There was no obvious site of attachment to the bowel wall, and there was no evidence of 
Fig. 2 Axial CT scan abdomen (before intravenous administration of contrast material) shows multiple fluidfilled and dilated loops of small bowel consistent with a mechanical small bowel obstruction
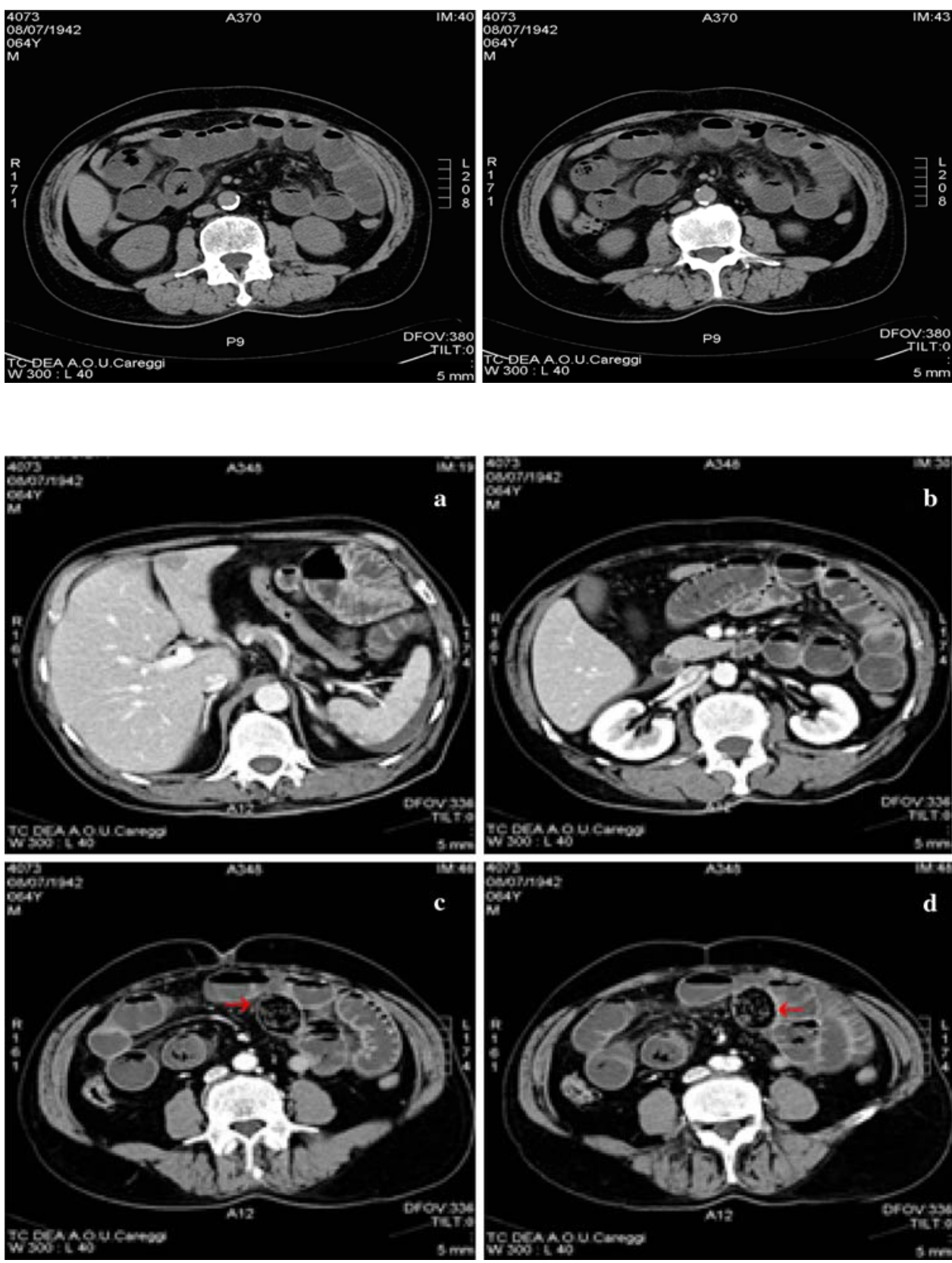

whorled appearance which we would expect to see in intussuception.

Dr. S. Vanni: The CT scan was nondiagnostic as to the cause of the obstruction, but did confirm the presence of small bowel obstruction. Do we have to go on with a conservative management? Did the patient's conditions evolve during this time? How did he feel after the initial management?

Dr. J. Attene: The patient quickly worsened despite the adequate fluid replacement. At physical examination, he showed mild disorientation, hyperventilation, warm extremities, mottled skin, tachycardia with a heart rate of
145 beats/min and a blood pressure of $230 / 120 \mathrm{mmHg}$. The body temperature was $37^{\circ} \mathrm{C}$. A new arterial blood gas revealed metabolic acidosis $(\mathrm{pH}$ 7.28) and even lower $\mathrm{HCO}_{3}{ }^{-}(15.8 \mathrm{mmol} / \mathrm{L})$. Serum lactate levels lowered (3.8 mmol/L) and hematocrit was 52\%. Clinical data hinted the presence of a systemic inflammatory response syndrome with signs of systemic hypoperfusion.

In relation to the persistently high values of blood pressure, labetalol and nitrate were intravenously administered to optimize cardiac preload, afterload and contractility, thus optimizing oxygen delivery to the tissues. Based on the signs of systemic hypoperfusion, the presence of blood in the fluid drained after nasogastric 
Table 2 Common cause of intestinal obstruction

\begin{tabular}{lll}
\hline Duodenum & Small bowel & Colon \\
\hline Stenosis & Adhesions & Carcinoma \\
Foreign body (bezoars) & Hernia & Fecal impaction \\
Stricture & Intussusception & Ulcerative colitis \\
Superior mesenteric artery & Limphoma & Volvulus \\
$\quad$ syndrome & & Diverticulitis \\
& & Intussusception \\
& & Pseudo-obstruction
\end{tabular}

Tinitinalli J, Kelen GD, Stapczynski JS (eds) (2004) Emergency medicine: a comprehensive study guide, 6th edn. McGraw-Hill, New York

tube placement and the history of alcohol assumption, we planned an upper gastrointestinal endoscopy, which however only showed edema of the gastric-stump.

The decision making for surgery at the initial surgery consult was difficult because the clinical presentation was typical but the plain film findings were equivocal. Moreover, while the CT scan showed signs of small bowel obstruction, they were mild and the etiology remained uncertain. Thus, the initial surgeon's decision delayed recommending surgical therapy for the patient.

Dr. G. Pepe: The differential diagnosis in this case mainly involves the common causes of intestinal obstruction (Table 2), among these small bowel obstruction (SBO) has the prevalent role.

The clinical presentation of SBO may be insidious and manifest as poor feedings. Diagnostic studies are merely ancillary and should not replace the clinical impression. In this case, we have laboratory findings that are consistent with a systemic inflammatory response syndrome (SIRS). We know that this syndrome can be seen like an early phase of septic shock, as a systemic inflammatory response to a variety of severe clinical insults. It is defined as the presence of two or more of the following features: (1) temperature greater than $38^{\circ} \mathrm{C}$ or less than $36^{\circ} \mathrm{C}$; (2) heart rate $>90$ beats/min; (3) respiratory rate $>20$ breaths/min; (4) white blood cell count $>12 \times 10^{9} / \mathrm{L},<4,000 \times 10^{9} / \mathrm{L}$, or $>10 \%$ immature (band) forms [2]. This syndrome is not a diagnosis, but it is a good indicator of outcome, and the SIRS criteria may be considered a crude stratification for patients with systemic inflammation. Our recent data has shown that patients with severe sepsis or septic shock represent $0.2 \%$ of all incoming patients to a tertiary care European ED, showing a $51 \%$ mortality rate at 28 -dayfollow up. Bedside prognostic indexes are useful in the recognition of patients at high risk for adverse short-term outcome [3]. In particular serum lactate $(>5 \mathrm{mmol} / \mathrm{L})$ is found to be significantly related to death within $24 \mathrm{~h}$ after admission. SIRS should be seen as an important clinical predictor of strangulated small bowel obstruction [4].

Dr. J. Attene: In our patient the urgency for surgical management was supported by several prognostic indexes. The sequential/sepsi organ failure assessment score (SOFA score), showed a score $=7$ (predominantly mild renal and bilirubin modifications). The APACHE II ("acute physiology and chronic health evaluation II"), another important ICU scoring system [5] showed 16 points with a calculated predicted death rate of $23.5 \%$.

Dr. S. Grifoni: What kind of considerations must we make in this case, with initial surgical uncertainly about operative or conservative management?

Dr. G. Pepe, Dr. J. Attene: A repeat surgical consult was planned, and after a careful clinical evaluation, it was decided to proceed with an exploratory laparotomy. At surgery, a $6 \times 12 \mathrm{~cm}$ stony hard mass firmly fixed within the lumen of the distal ileum, about $40 \mathrm{~cm}$ proximal to the ileocecal valve, was palpated. Enterotomy was performed, and a phytobezoar was removed. No underlying intestinal lesion was found.

The patient recovered uneventfully and was discharged 1 week later.

Dr. S. Vanni: There are four types of bezoars. Phytobezoars are the most common, and consists of fibrous material originating from vegetables and fruits, prune and persimmons. They contain a large amount of nondigestable fibers such as cellulose, hemicellulose, lignin and fruit tannins. It is well known that patients who have undergone a gastric operation are more prone to develop phytobezoars and their complications [6]. Trichobezoars are gastric concretions of hair fibers that usually develop in patients with psychosis. Pharmacobezoars consist of medication bezoars, which will adhere in bulk, such as cholestyramine, kayexalate resin, cavafate and antacids. Lactobezoars are milk curd secondary to infant formula, described in low birth weight neonates fed on highly concentrated formula within the first week of life [7,6] (Table 3).

The diagnosis of an obstructing bezoar is suggested in patients with previous gastric surgery presenting with intestinal obstruction. Nevertheless a SBO in these patients is more commonly due to adhesive bands or incisional hernias. Plain abdomen radiography is diagnostic in about $50-60 \%$ cases of SBO, equivocal in about $20-30 \%$, and normal or misleading in 10-20\%, regardless of etiology. An abdominal CT scan may then be useful in establishing the correct diagnosis. The advantages of a CT scan have given it an increasing role in evaluating patients with suspected SBO, mainly in patients in whom the clinical and plain film findings do not improve on nonoperative management, or in patients with acute abdominal 
Table 3 Etiology of bowel ischemia, necrosis, and perforation (total study group, $n=150) n(\%)$

Markogiannakis H et al (2007)

Acute mechanical bowel obstruction. World J

Gastroenterol January

$21 ; 13: 432-437$

\begin{tabular}{lcll}
\hline Cause & Ischemia $(n=21)$ & Necrosis $(n=14)$ & Perforation $(n=8)$ \\
\hline Hernia & $12(57.2 \%)$ & $6(42.8 \%)$ & $4(50 \%)$ \\
Large bowel cancer & $4(19.1 \%)$ & $3(21.4 \%)$ & $2(25 \%)$ \\
Adhesions & $3(14.3 \%)$ & $3(21.4 \%)$ & $2(25 \%)$ \\
Small bowel volvulus & $1(4.7 \%)$ & $1(7.2 \%)$ & $0(0 \%)$ \\
Sigmoid volvulus & $1(4.7 \%)$ & $1(7.2 \%)$ & $0(0 \%)$ \\
Small bowel tumor & $0(0 \%)$ & $0(0 \%)$ & $0(0 \%)$ \\
Retroperitoneal tumor & $0(0 \%)$ & $0(0 \%)$ & $0(0 \%)$ \\
Ovarian cystadenocarcinoma & $0(0 \%)$ & $0(0 \%)$ & $0(0 \%)$ \\
\hline
\end{tabular}

symptoms and abnormal, but nonspecific, plain film findings. The CT scan yields a high diagnostic accuracy in the detection of SBO, and in defining its severity and etiology. Recent studies confirm that a CT scan diagnosis of small bowel bezoar can be made by the presence of an intraluminal mass with mottled appearance and internal gas bubbles.

Dr. B. Dilaghi: Radiologists, in the emergency setting, should be alerted to the probability of a clinically unsuspected obstructing phytobezoar in the appropriate clinical setting of an adult man with previous gastric surgery or a suggestive dietary history, and should search for the diagnostic ovoid, mottled intraluminal mass at the site of the obstruction. Figure 3 shows this ambiguous finding [8].

\section{Discussion}

Intestinal obstructions account for $20 \%$ of surgical admission for an acute abdomen of which up to $80 \%$ are at the level of the small bowel [9]. Intra-abdominal adhesions account for the majority, approximately $70 \%$, of small bowel obstructions [10, 11]. The vast majority of the patients with adhesive obstruction have undergone prior abdominal operations [12]. Although an important share of these patients can be safely and effectively treated nonoperatively, particularly those with adhesive obstruction, a substantial portion require immediate operation. Strangulated obstruction requires emergency surgery, and early recognition is always life-saving since delay in treatment is an independent predictive factor of mortality. Traditionally the recognition of this condition is based on the presence of one or more of the classical signs of vascular compromise; continuous abdominal pain, fever, tachycardia, peritoneal signs on physical examination, leukocytosis and metabolic acidosis [13]. Moreover, the risk of strangulation is significantly higher in incarcerated hernias than other obstructive causes, such as adhesive obstruction. However, studies have shown that preoperative diagnosis of bowel strangulation cannot be made or excluded reliably by any known clinical, laboratory, or radiological parameter even if some factors, such as systemic inflammatory response syndrome, are proposed as predictor of strangulated small bowel obstruction [4]. Close and careful clinical evaluation, in conjunction with laboratory and radiological studies, is necessary for the decision for proper operative management. Nevertheless, if any uncertainty exists, prompt operative intervention is indicated [14]. In our case the hypothesis of small bowel obstruction due to adhesions was probable; as stated above, adhesions are the main cause of small bowel obstructions and our patient had undergone previous abdominal operations. Moreover on the CT scan, a diagnosis of adhesions is assumed when there is no identifiable lesion as was true in our case.

Another possible cause of intestinal obstruction in patients who have undergone a Billroth II resection of the stomach, is a fistula with obstruction of the gastrojejunostomy. The reattachment of the stomach with the small bowel is often done through the mesentery and recurrent ulcers of the gastrojejunostomy can cause a fistula with obstruction. This finding could be very hard to see at gastroscopy. Moreover, if the reattachment is antecolic, the long afferent limb can obstruct but appear normal or difficult to interpret at imaging studies. However, in our case the reattachment was mesocolic. It would be useful to know how the reattachment is made but this is not always possible. The decision to undertake emergency surgery was made in consideration of the clinical data that revealed diagnostic SIRS criteria, suggesting a high risk of strangulation. Our patient actually presented some of the classical signs of strangulation such as leukocytosis, metabolic acidosis and tachycardia with a systemic inflammatory response syndrome.

At surgery a phytobezoar, composed of vegetable fibers, was found impacted in the distal ileum and was successfully removed by enterotomy.

In contrast to adhesions, bezoars are an unusual cause of an acute abdomen due to small bowel obstruction. A bezoar is a concentration of ingested material within the gastrointestinal tract. Trichobezoars (concentrated balls of ingested hair) and phytobezoars (concentrations of poorly 
digested fibers, fruit seeds, and pulpy fruits) are the most common types of bezoars [15]. The latter are more common and are composed of cellulose, hemicellulose, lignin and fruit tannin. They most often develop in patients who have undergone gastric resection or ulcer surgery [16]. Studies have shown that more than $75 \%$ of patients who develop gastric or small bowel bezoars have a past history of surgery for peptic ulcer disease [17]. In accord with the literature, our patient had undergone gastric resection. Other predisposing factors are inadequate chewing, diabetic gastroparesis, and an excessive vegetarian diet [18]. Small bowel bezoars are often found in association with gastric bezoars. Primary small bowel bezoars are very rare and almost always present as intestinal obstruction, their most common complication [19]. Hence the clinical symptoms do not differ from those of small bowel obstruction resulting from other causes.

The radiology literature includes few reports of small bowel bezoars associated with small bowel obstruction, but the CT findings are characteristic, and this technique permits rapid and accurate diagnosis. The $\mathrm{CT}$ scanning of a small bowel bezoar demonstrates a well-defined oval, nonhomogeneous mass consisting of gas and soft tissue. The mass has a mottled appearance and internal gas bubbles because of air retained in its interstice, producing the characteristic looking of bezoar, allowing a specific preoperative diagnosis. However some authors report that this appearance may be similar to small bowel feces described in cases of severe stasis in patient with high-grade small bowel obstruction. Hence, especially in phytobezoars of soft tissue attenuation without air bubbles, it may be very difficult to make a definite diagnosis by CT scan [20]. In our case the CT scan did not reveal any characteristic pattern and an early diagnosis was not possible.

Bezoars usually become impacted in the narrowest portion of the small bowel that is $50-75 \mathrm{~cm}$ proximal to the ileocecal valve or at the valve itself [21]. Our patient's bezoar was located $40 \mathrm{~cm}$ proximal to the valve and no concurrent gastric bezoar was detected at endoscopy. Early surgery may be required for definitive therapy of small bowel bezoars because they can provoke ulceration, perforation, necrosis of the adjacent bowel, and even strangulation if diagnosis is delayed $[22,8]$.

Small bowel obstruction occurring from bezoar should be considered preoperativerly in patients with previous gastric surgery. Radiologists, in the emergency setting, should be alert to the probability of a clinically unsuspected obstructing phytobezoar in the appropriate clinical setting of an adult man with previous gastric surgery or a suggestive dietary history, and should search for the diagnostic ovoid, mottled intraluminal mass at the site of the obstruction.
In conclusion, early nasogastric decompression, aggressive fluid resuscitation, broad spectrum antibiotics and early surgical consultation are the mainstays of treatment of small and large bowel obstructions. The clinical diagnosis of bezoar induced small bowel obstruction, may be considered in patients with a previous gastric surgery presenting with intestinal obstruction.

Moreover, accurate early recognition of intestinal mechanical bowel obstruction is important to allow safe nonoperative management of carefully selected patients. Traditionally, nonoperative management is based on the absence of one or more of the classical signs of vascular compromise; continuous abdominal pain, fever, tachycardia, peritoneal signs on physical examination, leukocytosis, and metabolic acidosis. Close and careful clinical evaluation, in conjunction with laboratory and radiologic studies, are essential for the decision for proper management of these patients; if any uncertainty exists, prompt operative intervention is indicated. This case report emphasizes, though, that preoperative diagnosis of bowel strangulation cannot be made or excluded reliably by any known clinical, laboratory, or radiologic parameter, combinations of parameters, or by experienced clinical judgement. The SIRS evaluation, quickly and very simple, should be the warning sign for SBO. The most important question to be answered in the decision making for small bowel obstruction is: does this patient need an operation because of impending vascular compromise? It is not, and should not be: what is the cause of this obstruction.

\section{References}

1. American College of Emergency Physicians (2000) Clinical policy: critical issues for the initial evaluation and management of patients presenting with a chief complaint of nontraumatic acute abdominal pain. Ann Emerg Med 36:406-415

2. Bone RC, Balk RA, Cerra FB et al (1992) Definitions for sepsis and organ failure and guidelines for the use of innovative therapies in sepsis. The ACCP/SCCM Consensus Conference Committee. Chest 101:1644

3. Giannazzo G et al (2006) Prognostic indexes of septic syndrome in the emergency department. Intern Emerg Med 1:229-233

4. Tsumura H, Ichikawa T, Hiyama E, Muratami Y, Sueda T (2004) Systemic inflammatory response sindrome (SIRS) as a predictor of strangulated small bowel obstruction. Hepatogastroenterology 51:1393-1396

5. Knaus WA, Draper EA, Wagner DP, Zimmerman JE (1985) Prognosis in acute organ-system failure. Ann Surg 202(6):685693

6. Moshe R (1998) Phytobezoar: a rare cause of intestinal obstruction. Dig Surg 15:52-54

7. Andrus CH, Ponsky JL (1988) Bezoars: classification, pathophysiology and treatment. Am J Gastroenterol 83:476-478

8. Quiroga S, Alvarez-Castells A, Sebastia MC, Palisa E, Barluenga E (1997) Small bowel obstruction secondary to bezoars: CT diagnosis. Abdom Imaging 22:315-317 
9. Welch JP (1990) General consideration and mortality in bowel obstruction. In: Welch JP (ed) Bowel obstruction differential diagnosis and clinical management. Saunders, Philadelphia, pp 59-95

10. Menzies D (1992) Peritoneal adhesions; incidence, cause and prevention. Surg Annu 24:29-45

11. Miller G, Boman J, Shrier I, Gordon PH (2000) Etiology of small bowel obstruction. Am J Surg 180:33-36

12. Kossi J, Salminen P, Laato M (2004) The epidemiology and treatment patterns of postoperative adhesion induced intestinal obstruction in Varsinais-Suomi Hospital District. Scand J Surg 93:68-72

13. Sarr MG, Bulkley GB, Zuidema GD (1983) Preoperative recognition of intestinal strangulation obstruction. Prospective evaluation of diagnostic capability. Am J Surg 145:176-376

14. Mucha P Jr (1987) Small intestinal obstruction. Surg Clin North Am 67:597-620

15. Zissin R, Osadchy A, Gutman V, Rathaus V, Shapiro-Feinberg M, Gayer G (2004) CT findings in patients with small bowel obstruction due to phytobezoar. Emerg Radiol 10:197-200
16. Chisholm EM, Leong HT, Chung SC et al (1992) Phytobezoars: an uncommon cause of small bowel obstruction. Ann R Coll Surg Engl 74:342-344

17. Robles R, Parrilla P, Escamilla C et al (1994) Gastrointestinal bezoars. Br J Surg 81:1000-1001

18. Escamilla C, Robles-Campos R, Parrilla-Paricio P, Lujan-Mompean J, Liron Ruiz R, Torralba-Martinez JA (1994) Intestinal obstruction and bezoars. J Am Coll Surg 179:285-288

19. Saeed ZA, Rabassa AA, Anand BS (1995) An endoscopic method for removal of duodenal phytobezoars. Gastrointes Endosc 41:106-108

20. Frager DH, Baer JW (1995) Role of TC in evaluating patients with small bowel obstruction. Semin Ultrasound CT MR 16:127140

21. Wang PY, Skargard DE, Barker J (1996) Carpet bezoar obstruction. J Pediatr Surg 31:1691-1963

22. Verstandig AG, Klin B, Bloom RA, Hadas I, Libson E (1989) Small bowel phytobezoars: detection with radiography. Radiology 172:705-707 